\title{
Simple avulsion of onychogryphotic toenails: a justifiable treatment?
}

\author{
J.D. Greig, ${ }^{*}$ J.H. Anderson, A.J. Ireland, J.R. Anderson. \\ Departments of Surgery, Hairmyres Hospital, East Kilbride and The Royal Infirmary, Glasgow, UK.
}

\begin{abstract}
Summary: Twenty-nine consecutive patients with onychogryphotic toenails in whom conservative treatment had failed, were entered into the study over a one year period and followed-up prospectively for a further year. All patients were treated by simple nail avulsion. The incidence of recurrence was 27 out of 29 patients (93\%); only five of these were symptomatic (17\%). Only two patients required further surgery. We conclude that simple avulsion of onychogryphotic toenails offers symptomatic relief in the majority of patients, particularly in this elderly population in whom the use of a tourniquet may be contraindicated.
\end{abstract}

\section{Introduction}

The management of onychogryphotic toenails and the outcome following surgery are variable and usually considered along with that of ingrowing toenails. A comparison of nine collected studies has shown that the results of surgery are procedure-, surgeon- and study-dependent. ${ }^{1}$ Onychogryphosis is a distinct entity, differing from ingrowing toenails in pathology, sex distribution and the age of the population affected. There is a paucity of information in the literature regarding the natural history and the management of this condition. ${ }^{2}$ The aim of the present study was to assess the efficacy of simple nail avulsion without ablation in treating onychogryphotic toenails.

\section{Patients and methods}

Consecutive patients with onychogryphotic toenails in whom conservative treatment had failed, and who were referred to the departments of general surgery at Hairmyres Hospital, and Glasgow Royal Infirmary, between 1st August 1986 and 31st July 1987 were included in the study. Patients with ingrowing toenails (onychocryptosis) were excluded. All patients were treated by simple nail avulsion. The operations were performed by one of three surgical registrars.

Correspondence: J.R. Anderson, F.R.C.S., University Department of Surgery, Royal Infirmary, Alexandra Parade, Glasgow G31 2ER, UK.

*Present address: University Department of Surgery, Royal Infirmary of Edinburgh, Laurieston Place, Edinburgh EH3 9YW.

Accepted: 26 May 1989
Peripheral pulses and the distal circulation were checked before and after the procedure. The affected toe was anaesthetized by a digital block using $5-10 \mathrm{mb}$ of $0.5 \%$ marcaine plain. Bacteriological specimens taken from infected or moist nails were cultured fog fungi and bacteria. The whole nail was removed b undermining the nail plate and avulsing it with a pais of artery forceps. Any surrounding granulation 7 infected tissue was curetted.

Dressings were changed at 3 days and subsequent post-operative care was provided by the general practitioner. Patients were assessed 2 weeks later for early complications which were documented and bacteriological cultures were taken where appropriate. Following discharge, patients were advised on wound care, footwear and hygiene.

The patients were recalled for follow-up one year later at a toenail clinic and the results of their treatment were documented on a standard proforma by an independent assessor.

\section{Results}

Thirty patients entered the study, one of whom was later excluded because he underwent a below knee amputation for severe peripheral vascular disease, leaving 29 patients in whom the results of surgery were assessed.

There were 12 males and 17 females with a mean age of 58 years (median age 61 years) (range: $3-83$ ). The left side was affected in 16 patients and the right side in 13 patients. All toenails had caused bilateral nail-fold symptoms for more than 4 months prior to presentation. 
Two patients (7\%) had pre-operative fungal infections. There were no infections post-operatively. Only two patients (7\%) had no recurrence. Recurrence took the form of onychogryphosis (22 patients) or nail dystrophy ( 5 patients). The mean time to recurrence was 6.7 months (range: $2-12$ ).

Of the 27 patients with a recurrence, five (17\%) patients were symptomatic, two required avulsion and phenolization of the germinal matrix and the other three required chiropody. The other 22 patients $(76 \%)$ were asymptomatic. Overall, 24 patients $(83 \%)$ were satisfied with the results of their treatment at one year. Four of these patients complained of discomfort associated with the digital anaesthetic block and should further surgery be necessary, they would decline.

\section{Discussion}

The results of this study show that, as expected there was a high recurrence rate $(93 \%)$. However, the majority of recurrent onychogryphotic nails were improved after simple avulsion and three-quarters of the patients were asymptomatic one year later. This study also emphasizes that, with the exception of a 3 year old boy who developed an onychogryphotic nail secondary to trauma, this condition principally affects the elderly population, and males and females are affected in similar numbers. Onychogryphosis should therefore be considered separately from ingrowing toenails.

\section{References}

1. Sykes, P.A Ingrowing toenails: time for critical appraisal? $J R$ Coll Surg Edin 1986, 31: 300-304.

2. Greig, J.D. The results of treatment of onychogryphotic toenails in a general hospital. Chiropodist 1988, 43: 35-36.
The outcome of ablative procedures is variable with recurrence rates of between 0 and $85 \%$ being reported, although these studies also included ingrowing toenails. ${ }^{1}$ The only study to our knowledge which has attempted to analyse critically the results of three treatments specifically for onychogryphosis was a retrospective 3 year audit. ${ }^{2}$ The recurrence rates for avulsion, avulsion and phenolization, and nail bed excision were 82,44 , and $83 \%$ respectively. These rates were reduced to 59,19 and $83 \%$ respectively when only symptomatic recurrence was considered. The incidence of symptomatic recurrence in the avulsion group was much higher $(59 \%)$ than in the present study $(17 \%)$, but our follow-up was only 12 months compared with a mean follow-up of 27 months in the previous report.

Since most recurrences are asymptomatic, simple avulsion of onychogryphosis offers a reasonable outcome, particularly where there may be a contraindication to the use of a tourniquet. In those symptomatic recurrences which fail to respond to chiropody, consideration should be given to germinal matrix phenolization. Further long-term prospective studies are required to assess whether recurrences after simple avulsion become symptomatic with the passage of time.

\section{Acknowledgements}

The authors wish to thank the medical and nursing staff in Hairmyres Hospital and Glasgow Royal Infirmary for their cooperation and help in supporting this study. 